11,05

\title{
Фазовые переходы в трехмерной слабо разбавленной модели Поттса с $q=5$
}

\author{
(c) А.К. Муртазаев ${ }^{1}$, А.Б. Бабаев ${ }^{1,2}$ \\ ${ }^{1}$ Институт фозики им. Х.И. Амирханова Дагестанского федерального исследовательского центра РАН, \\ Махачкала, Россия \\ 2 Дагестанский фредеральный исследовательский центр РАН, \\ Махачкала, Россия \\ E-mail: b_albert78@mail.ru
}

Поступила в Редакцию 6 марта 2021 г.

В окончательной редакции 6 марта 2021 г.

Принята к публикации 5 июня 2021 г.

Методом компьютерного моделирования проведено исследование фазовых переходов в трехмерной слабо разбавленной модели Поттса с числом состояний спина $q=5$. Рассмотрены системы с линейными размерами $L \times L \times L=N, L=10-40$ при концентрации спинов $p=1.00,0.90$. Полученные численные данные свидетельствуют о том, что внесение незначительного беспорядка в виде немагнитных примесей $(p=0.90)$ в трехмерной модели Поттса с $q=5$ не существенен для фазового перехода первого рода.

Ключевые слова: примесь, модель Поттса, метод Монте-Карло, термодинамические параметры, критические явления.

DOI: 10.21883/FTT.2021.10.51417.047

\section{1. Введение}

Вектор исследования фазовых переходов (ФП) и критических свойств магнитных систем под воздействием беспорядка, сместился в сторону применения вычислительных методов. Это обусловлено тем, что моделирование с использованием методов Монте-Карло (МК) позволяет изучать более реалистичные модели и учитывать усложняющие факторы, всегда присутствующие в реальных материалах [1-5]. Этому способствуют и серьезно возросшие вычислительные возможности современных компьютеров, и множество новейших и мощных алгоритмов, специально разработанных для использования в этой области. Поэтому метод Монте-Карло с использованием современных алгоритмов зарекомендовал себя как мощный инструмент для систематического изучения магнитных систем, особенно при изучении фазовых переходов и магнитных явлений [1].

Одной из моделей применяемых для описания реальных физических систем, является модель Поттса. Очевидно, что решеточная структура данной модели изоморфна многим таким системам как: слоистый магнетик, аэрогели, пленки жидкого гелия, сверхпроводящие пленки и т.д. [6]. Изучение этой модели в присутствии немагнитного беспорядка позволяет определить точные значения концентраций немагнитных примесей $c$, $c=1-p$, где $p-$ концентрация спинов, при которых в рассматриваемой системе может происходить смена рода фазового перехода. Определение точных значений концентраций немагнитных примесей имеет большое значение при создании различных новых магнитных материалов, а также при изучении высокотемпературных сверхпроводников, образующих при замещении небольшого количества магнитных атомов La немагнитными атомами стронция $\mathrm{Sr}$ в антиферромагнитном диэлектрике $\mathrm{LaCuO}_{4}$ [7]. Кроме того, к настоящему времени остается открытым вопрос о том, что фазовые переходы первого рода наблюдаются ли в присутствии беспорядка? Выяснение ответа на этот вопрос является главной целью настоящей работы.

\section{2. Модель и метод исследования}

В работе рассматривается трехмерная слабо разбавленная модель Поттса с числом состояний спина $q=5$. При построении такой модели необходимо иметь в виду следующие особенности: в узлах кубической решетки расположены спины $\mathrm{Si}$, которые могут находиться в одном из $q \geq 2$-состояний и немагнитные примеси (вакансии); немагнитные примеси распределены случайно и фиксированы (канонический способ) на различных узлах решетки (quenched disorder); энергия связи между двумя узлами равна нулю, если они находятся в разных состояниях (безразлично, в каких именно) или же, если хотя бы в одном узле находится немагнитный атом, и равна $|J|$, если взаимодействующие узлы находятся в одинаковых состояниях (опять же, все равно в каких именно). С учетом этих особенностей микроскопический гамильтониан такой системы может быть, представлен в виде [6]:

$$
H=-\frac{1}{2} J \sum_{i, j} \rho_{i} \rho_{j} \delta\left(S_{i}, S_{j}\right), \quad S_{1}=1,2,3,4,5,
$$


где

$$
\delta\left(S_{i}, S_{j}\right)=\left\{\begin{array}{l}
1, \\
0, \text { если } S_{i}=S_{j}, \\
\text { если } S_{i} \neq S_{j}
\end{array}\right.
$$

и

$\rho_{i}=\left\{\begin{array}{l}1, \text { если в узле расположен спин, } \\ 0, \text { если в узле расположена немагнитная примесь. }\end{array}\right.$

Исследования проводились на основе высокоэффективного кластерного алгоритма Вольфа [8]. Методика реализации этого алгоритма приведена в работе [9]. Расчеты проводились для систем с периодическими граничными условиями при концентрациях спинов $p=1.00 ; 0.90$. Исследовались системы с линейными размерами $L \times L \times L=N, L=10 \div 40$. Начальные конфигурации задавались таким образом, чтобы все спины были упорядочены вдоль оси $Z$. Для вывода системы в равновесное состояние отсекался неравновесный участок $\tau_{0}$ длиной для системы с линейными размерами $L$. Этот неравновесный участок отбрасывали. Затем усреднение проводилось по участку марковской цепи длиной $\tau=160 \tau_{0}$. Для самой большой системы $L=40$, $\tau_{0}=2.3 \cdot 10^{3} \mathrm{MК} \mathrm{шагов/спин.} \mathrm{Кроме} \mathrm{того,} \mathrm{проводилось}$ усреднение по различным начальным конфигурациям. В случае $p=1.0$ для усреднения использовалось 10 начальных конфигураций. Для слабо разбавленных систем с концентрацией спинов $p=0.90$ осуществлялось конфигурационное усреднение по 2000 различным конфигурациям, причем для каждой примесной конфигурации выполнялось усреднение по длине цепи $\tau=160 \tau_{0}$.

\section{3. Результаты численного эксперимента}

Для наблюдения за температурным ходом энергии $U$, намагниченности $m_{F}$, теплоемкости $C$ и восприимчивости $\chi$ нами использовались следующие соотношения $[10,11]$ :

$$
\begin{gathered}
U=[\langle U\rangle]=\frac{1}{N}[\langle H\rangle], \\
m_{F}=\frac{\left[q\left(\frac{N_{\max }}{N}\right)-1\right]}{q-1}, \\
C=\left(C K^{2}\right)\left\lfloor\left(\left\langle U^{2}\right\rangle-\langle U\rangle^{2}\right)\right\rfloor, \\
\chi=(N K)\left\lfloor\left(\left\langle m^{2}\right\rangle-\langle m\rangle^{2}\right)\right\rfloor,
\end{gathered}
$$

где $K=|J| / k_{\mathrm{B}} T, N_{\max }=\max \left\{N_{1}, N_{2}, N_{3}, N_{4}, N_{5}\right\}, N_{i}-$ число спинов в состоянии с $q=i, N=p L^{3}$ - число магнитных узлов, угловые скобки означают термодинамическое усреднение, квадратные скобки означают усреднение по примесным конфигурациям.

На рис. 1 представлены температурные зависимости энергии $U$ для модели Поттса с числом состояний спина $q=5$ при концентрации спинов $p=1.0$ и $p=0.9$ для спиновых систем с линейными размерами $L=40$. Здесь и далее на всех рисунках погрешность данных не

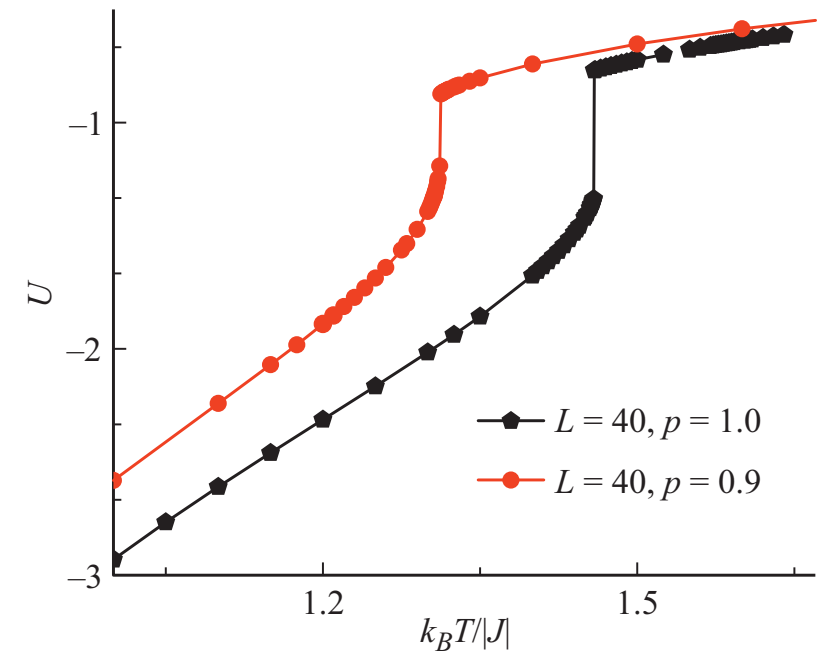

Рис. 1. Температурная зависимость энергии для модели Поттса.

превосходит размеров символов, используемых для обозначения зависимости. Как видно из рис. 1 температурные зависимости энергии для рассмотренных значений концентраций $p$ демонстрируют поведение характерное для фазового перехода первого рода (в точке фазового перехода $T_{l}(p)$ проявляется отчетливый скачок энергии). Как видно из рисунка наличие слабого беспорядка в виде немагнитных примесей с концентрацией $c=0.1$ $(p=1-c)$ не подавляет фазовый переход первого рода.

На рис. 2, 3 и 4 представлены характерные зависимости теплоемкости $C$, восприимчивости $\chi$ и намагниченности $m_{F}$ для систем с разными линейными размерами $L$ при концентрации спинов $p=1.0$ и $p=0.9$. Как видно из рис. 2 и 3 в зависимостях теплоемкости $C$ и восприимчивости $\chi$ от температуры $T$ для всех исследуемых нами однородных и слабо разбавленных систем описываемых трехмерной моделью Поттса с $q=5$ в точке фазового перехода проявляются „всплески“, которые характерны для фазового перехода первого рода. Температурные зависимости намагниченности $m_{F}$ при $p=1.0$ и $p=0.9$ испытывают скачок в области фазового перехода (см. рис. 4).

При моделировании системы, для определения температуры фазового перехода $T_{l}(p)$ часто используют метод кумулянтов Биндера четвертого порядка [12]

$$
\begin{gathered}
V_{L}(T, p)=1-\frac{\left\langle E^{4}\right\rangle_{L}}{3\left\langle E^{2}\right\rangle_{L}^{2}}, \\
U_{L}(T, p)=1-\frac{\left\langle m^{4}(T, p ; L)\right\rangle_{L}}{3\left\langle m^{2}(T, p ; L)\right\rangle_{L}^{2}},
\end{gathered}
$$

где $E-$ энергия и $m_{F}-$ намагниченность системы с линейным размером $L$. Выражения (6) и (7) позволяют определить $T_{l}(p)$ с большой точностью в фазовых переходах первого и второго рода соответственно. Так же данный метод, хорошо зарекомендовал себя и при 


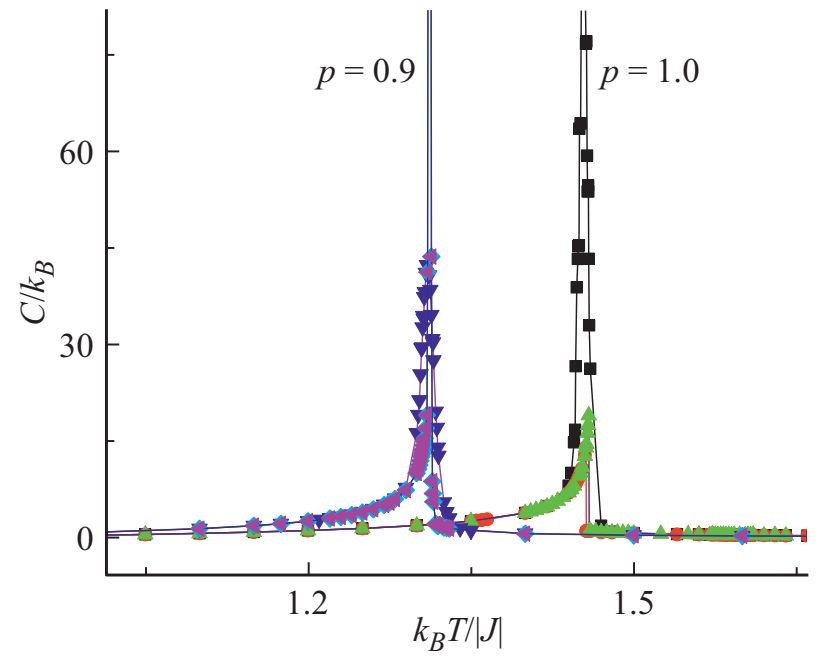

Рис. 2. Температурная зависимость теплоемкости для модели Поттса.

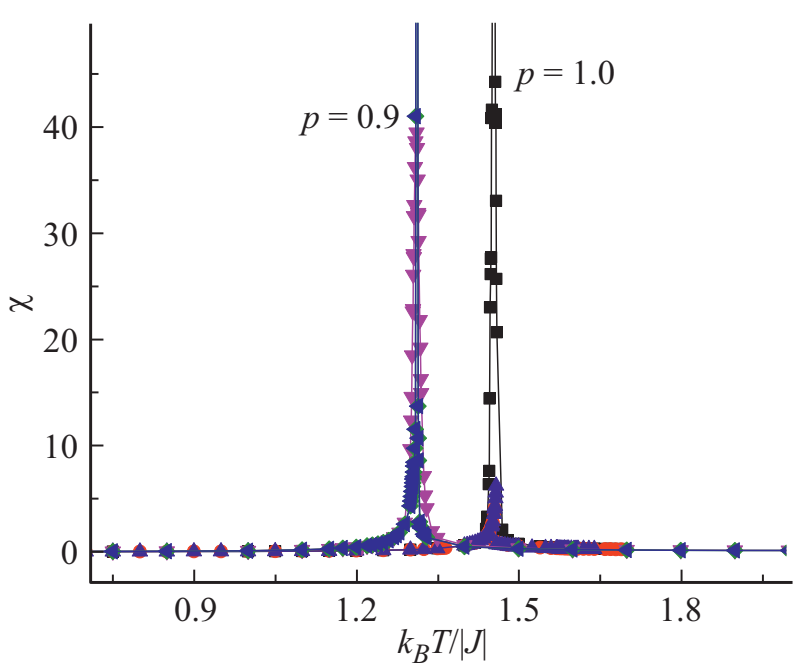

Рис. 3. Температурная зависимость восприимчивости для модели Поттса.

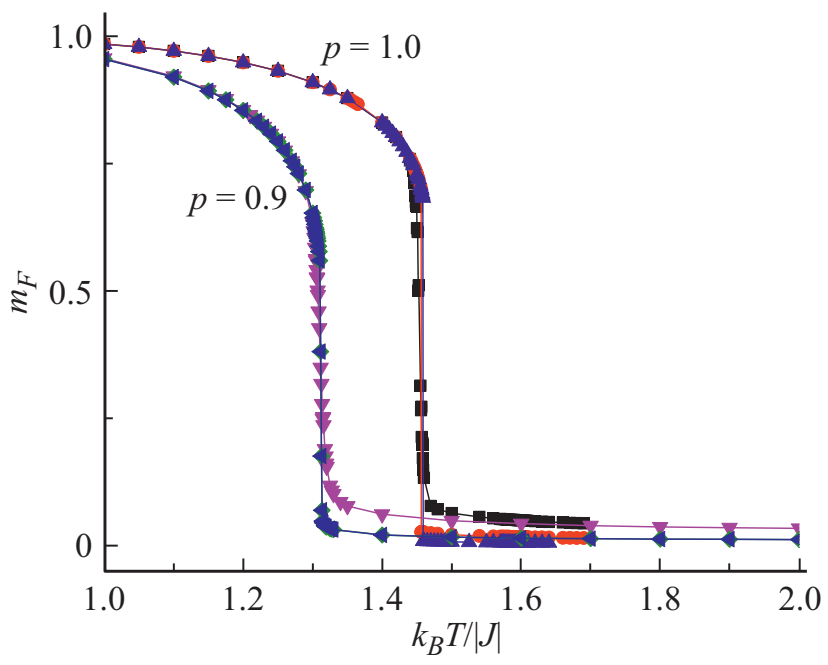

Рис. 4. Температурная зависимость намагниченности для модели Поттса.

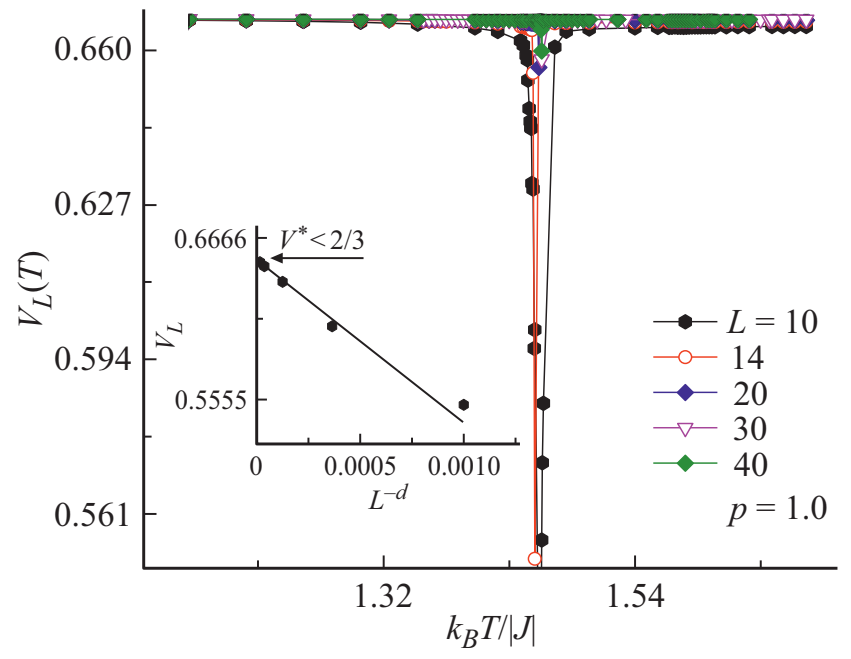

Рис. 5. Температурная зависимость кумулянтов Биндера $V_{L}(T)$ для чистой неразбавленной $(p=1.0)$ модели Поттса.

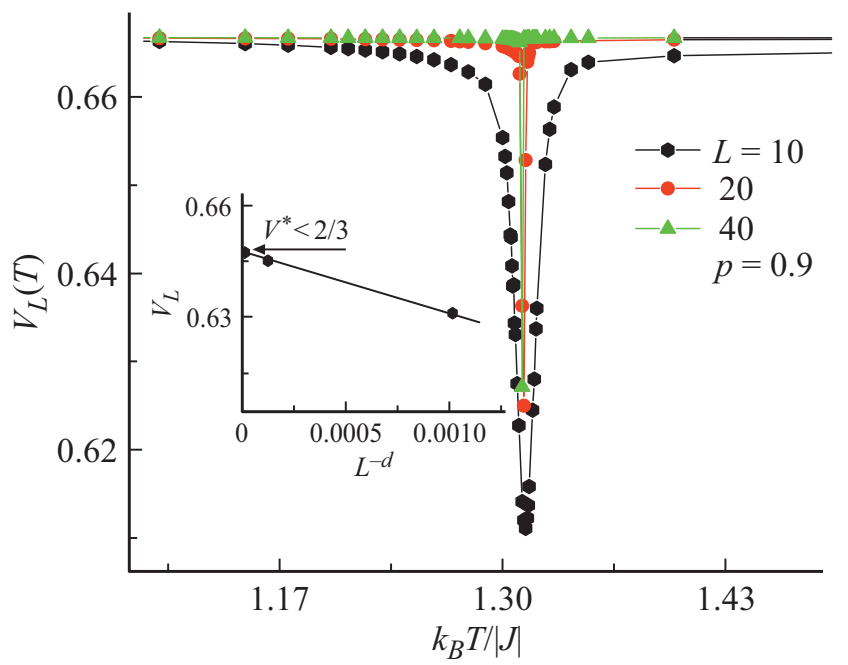

Рис. 6. Температурная зависимость кумулянтов Биндера $V_{L}(T)$ для слабо разбавленной $(P=0.9)$ модели Поттса.

определении рода ФП. Анализ численных данных с применением этого метода представлены в работах [13-15]. Отличительные черты характерные для ФП [16]: для ФП первого рода характерно то, что усредненная величина $V_{L}(T, p)$ стремится к некоторому нетривиальному значению $V^{*}$ согласно выражению

$$
V(T, p)=V^{*}+b L^{-d}
$$

при $L \rightarrow \infty$ и $T=T_{i}(L)$, где $V^{*}$ отлична от $2 / 3$, что и продемонстрировано на рис. 5 и 6 соответственно для модели Поттса с $q=5$ при концентрации спинов $p=1.0$ и $p=0.9$. Кроме того, при фазовых переходах первого рода минимальная величина $U_{l, \min }\left(T=T_{\min }, p\right)$ расходится $U_{L, \min }\left(T=T_{\min }, p\right) \rightarrow-\infty$, при $L \rightarrow \infty$, (см. рис. 7). Аналогичное поведение наблюдалось при концентрации спинов $p=1.0$. Как видно из полученных данных для модели Поттса с $q=5$ внесение незначительного количества примесей концентрацией $c=0.1(c=1-p)$ не 


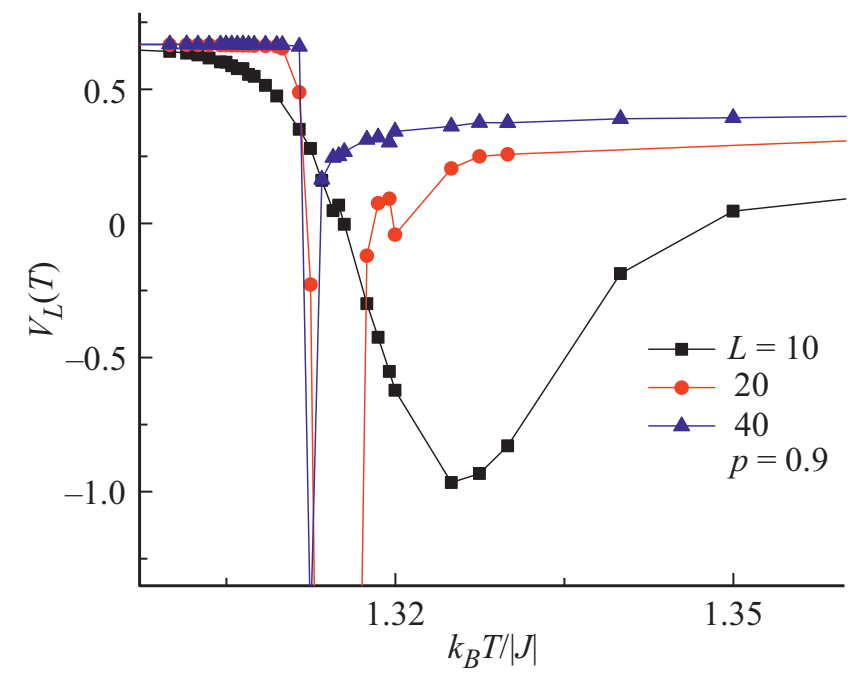

Рис. 7. Температурная зависимость кумулянтов Биндера $U_{L}(T)$ для слабо разбавленной $(p=0.9)$ модели Поттса.

существенна для смены рода ФП. В то же время для этой модели при $q=3$ в работе [17] было показано, что наличие слабого беспорядка в виде немагнитных примесей концентрацией $c=0.05$ достаточно для смены фазового перехода первого рода на фазовый переход второго рода. Для случая с $q=4$ [18] такая смена рода фазового перехода в слабо разбавленном режиме не наблюдалась. По-видимому, вмороженный беспорядок на разных трехмерных моделях Поттса сказывается по-разному. Следует также отметить, что полученные данные для неразбавленной $(p=1.0)$ трехмерной модели Поттса $\mathrm{c}$ $q=5$ с применением метода Монте-Карло в настоящей работе находятся в хорошем согласии с недавними данными полученными на основе метода нейронных сетей [19]. Метод нейронных сетей как метод машинного обучения, идеи которого изложены в работе [20] может стать альтернативой традиционному методу МонтеКарло. Одним из ограничений стандартного метода машинного обучения является знание точного значения критической температуры до проведения расчетов. В настоящее время разрабатываются другие схемы метода машинного обучения, в которых не столь важно знание наперед точного значения критической температуры.

В отличии от трехмерных решеточных моделей для низкоразмерных моделей $(d=2)$ Поттса с числом состояний спина $q=3, q=4$ наличие слабого беспорядка сказывается на критическое поведение (см. работы $[21,22])$, а в случае моделей Поттса с $q \geq 5$ вмороженный беспорядок существенен при фазовых переходах первого рода и всегда приводит к смене фазового перехода первого рода на фазовый переход второго рода $[23,24]$.

\section{4. Заключение}

В настоящей работе исследованы фазовые переходы в трехмерной слабо разбавленной ферромагнитной моде- ли Поттса с числом состояний спина $q=5$ на простой кубической решетке. Полученные данные в результате наших исследований свидетельствуют о том, что в трехмерной ферромагнитной модели Поттса с $q=5$ на простой кубической решетке наблюдается фазовый переход первого рода в соответствии с предсказаниями аналитических теорий [6] и внесение слабого беспорядка $(p=0.90)$ в виде вмороженных немагнитных примесей в рассматриваемую модель не приводит к подавлению фазового перехода первого рода.

\section{Конфликт интересов}

Авторы заявляют,что у них нет конфликта интересов.

\section{Список литературы}

[1] Л.Н. Щур. УФН 182, 7, 787 (2012).

[2] O. Vasilyev, B. Berche, M. Dudka, Yu. Holovatch. Phys. Rev. E 92, 042118 (2015).

[3] D.P. Landau, K. Binder. A guide to Monte Carlo simulations in statistical physics. Cambridge university press ( 2014).

[4] A.K. Murtazaev, A.B. Babaev. Mater.Lett. 258, 126771 (2020).

[5] A.K. Murtazaev, A.B. Babaev, G.Y. Ataeva. J. Magn. Magn. Mater. 440, 101 (2017).

[6] F.Y. Wu. Exactly Solved Models: A Journey in Statistical Mechanics. World Scientific, London( 2009).

[7] L.A. Fernandez, A. Gordillo-Guerrero, V. Martin-Mayor, J.J. Ruiz-Lorenzo. Phys. Rev. B 86, 184428 (2012).

[8] U. Wolff. Phys. Lett. 62, 361 (1989).

[9] А.Б. Бабаев, А.К. Муртазаев. Математическое моделирование 30, 12, 55 (2018).

[10] Р. Бекстер. Точнорешаемые модели в статистической механике. Мир, М. (1985). 488 с.

[11] P. Peczac, A.M. Ferrenberg, D.P. Landau. Phys. Rev. B 43, 6087 (1991).

[12] K. Eichhorn, K. Binder. J. Phys.: Condens. Matter 8, 5209 (1996).

[13] A.K. Murtazaev, A.B. Babaev, G.Y. Ataeva. EPJ Web of Conferences 185, 11001 (2018).

[14] А.К. Муртазаев, А.Б. Бабаев, Г.Я. Атаева. ФТТ 57, 7, 1410 (2015).

[15] А.Б. Бабаев, Т.Р. Ризванова, А.К. Муртазаев. ФТТ 59, 12, 2416 (2017).

[16] D. Loison, K.D. Schotte. Eur. Phys. J. B 5, 735 (1998).

[17] А.Б. Бабаев, А.К. Муртазаев. Письма в ЖЭТФ 105, 6, 363 (2017).

[18] А.К. Муртазаев, А.Б. Бабаев. Письма в ЖЭТФ 99, 618 (2014).

[19] D.R. Tan, C.D. Li, W.P. Zhu, F.J. Jiang. New J. Phys. 22, 063016 (2020).

[20] C.D. Li, D.R. Tan, F.J. Jiang. Ann. Phys. 391, 312 (2018).

[21] А.Б. Бабаев, А.К. Муртазаев, ФТТ 62, 5, 757 (2020).

[22] A.K. Murtazaev, A.B. Babaev Mater. Lett. 238, 321 (2019).

[23] А.Б. Бабаев, А.К. Муртазаев. ФТТ 62, 7, 1088 (2020).

[24] Xiaofeng Qian, Youjin Deng, Henk W.J. Blöte. Phys. Rev. E 72, 056132 (2005)

Редактор Т.Н. Василевская 